\title{
Vermittlung des Weiterverkaufs des sog. Borghorster Stiftskreuzes als Begünstigung
}

LG Münster (Große Strafkammer) Urteil vom 5.5.2016 - 3 Kls - 200 Js 10/16 - 11/16

\section{ECLI:}

ECLI:DE:LGMS:2017:0505.3KLS200JS10.16.11.00

\section{Tenor:}

- Der Angeklagte wird wegen Begünstigung zu einer Freiheitsstrafe von einem Jahr neun Monaten verurteilt, deren Vollstreckung zur Bewährung ausgesetzt wird.

Im Übrigen wird der Angeklagte freigesprochen.

Der Angeklagte hat die Kosten des Verfahrens zu tragen, soweit er verurteilt wurde; soweit er freigesprochen wurde, trägt die Staatskasse die Kosten des Verfahrens und die notwendigen Auslagen des Angeklagten.

Von einer Entscheidung über den Adhäsionsantrag des Bistums Münster vom 7.3.2017 wird abgesehen.

Die durch das Adhäsionsverfahren entstandenen gerichtlichen Auslagen werden der Staatskasse auferlegt. Die sonstigen durch dieses Verfahren entstandenen Auslagen trägt jeder Beteiligte selbst.

Angewendete Vorschriften: $§ 257$ Abs. 1, 56 StGB.

\section{[1] Gründe:}

[2] (abgekürzt gemäß § 267 Abs. 4 StPO)

[3] I.

[4] Der Angeklagte wurde entweder im Libanon oder in der Türkei an der syrischen Grenze geboren. Er hat sieben Geschwister. 1978 floh er mit seiner Familie nach C1, als er vier bis fünf Jahre alt war. Sein genaues Geburtsjahr ist ihm nicht bekannt. Die Papiere wurden verbrannt. Die Familie zog in das Asylbewerberlager "V". Der Angeklagte besuchte bis 1986 in V eine Sonderschule. Im selben Jahr verließ sein Vater die Familie, nachdem auf inn geschossen worden war. Seine Mutter besorgte einen gefälschten Pass, flüchtete mit den weiteren Familienmitgliedern nach C2 und stellte dort einen Asylantrag. Seitdem lebt der Angeklagte in C2. Sein Vater verstarb im Jahr 1999.

[5] Der Angeklagte hat keinen Schulabschluss. Lesen und schreiben lernte er nach eigenen Angaben nicht. Später nahm er an einer ABM-Maßnahme teil. Seit 1992/93 hat er eine Arbeitserlaubnis. Er arbeitete als Lagerist und Packer, wurde kriminell und verbüßte auch mehrjährige Haftstrafen.
[6] Zwischenzeitlich betätigte er sich als Türsteher in C2 an der T1 im "M1". Vor der Festnahme im hiesigen Verfahren arbeitete er als Packer und verdiente durchschnittlich etwa 1000 Euro netto.

[7] 1999 heiratete er. Seine Ehe ist gescheitert. Er hat eine Lebensgefährtin, mit der er sich verlobt hat, und fünf Kinder. Der älteste Sohn ist 17 Jahre alt und lebt seit zwei Jahren beim Angeklagten.

[8] Probleme wegen Alkohols oder Drogenkonsums hat der Angeklagte nicht. Schulden bestehen nur hinsichtlich Gerichtskosten aus einem anderen Verfahren. Insoweit wurde ein Vergleich mit dem Rechtspfleger erzielt.

[9] Der Angeklagte ist bereits strafrechtlich in Erscheinung getreten. Ausweislich des Auszugs aus dem Bundeszentralregister vom 12.10.2016, der insgesamt 16 Eintragungen enthält, wurde er unter anderem bereits wie folgt verurteilt:

[10] Am 00.00.0000 verurteilte ihn das Landgericht P1 - KLs 000 Js 00000/00 - wegen unerlaubter Einfuhr von Betäubungsmitteln in nicht geringer Menge zu einer Freiheitsstrafe von drei Jahren neun Monaten. Nachdem der Angeklagte zwei Drittel der Strafe verbüßt hatte, wurde ein Strafrest bis zum 5.3.2001 zur Bewährung ausgesetzt.

[11] Am 00.00.00000 verurteilte ihn das Landgericht C2 - 00 KLs 000 Js 00000/00 - wegen unerlaubten Handels mit Betäubungsmitteln in nicht geringer Menge in drei Fällen, davon in zwei Fällen in Tateinheit mit Beihilfe zur unerlaubten Einfuhr von Betäubungsmitteln in nicht geringer Menge zu einer Gesamtfreiheitsstrafe von vier Jahren zwei Monaten. Ein Strafrest wurde zur Bewährung ausgesetzt bis zum 12.12.2009 und letztlich erlassen mit Wirkung vom 8.4.2010.

[12] II.

[13] 1.

[14] Die drei miteinander gut und seit Jahren bekannten anderweitig Verurteilten T2 (ein Neffe des Angeklagten), U1 und N1 fuhren am 00.00.0000 zusammen mit einer bislang unbekannten vierten Person mit einem dunklen Pkw, Marke Mercedes Benz, C-Klasse Kombi, mit dem amtlichen Kennzeichen 00-00 000 zunächst zur BAB-Raststätte X1 unweit von ihrem Wohnort C2 und verließen dort das Fahrzeug. Nach dem Halt fuhren sie weiter zur Tankstelle $\mathrm{M} 2$ in B in der Nähe von Münster und von dort weiter zu der nahegelegenen T5-Tankstelle 
in B, vermutlich, um dort jeweils Diebstähle zu begehen. Anschließend fuhren sie mit dem Pkw nach $\mathrm{T} 2$ und hielten einige Minuten vor 13.24 Uhr am Kirchplatz an der O-Kirche, weil sie beabsichtigten, gemeinsam aus der Kirche das sogenannte „Borghorster Stiftskreuz" zu entwenden.

[15] T2, U1 und N1 stiegen aus dem Fahrzeug und liefen in die O-Kirche. Der Fahrer fuhr zunächst weiter. In der Kirche öffnete U1 gewaltsam gegen 13.24 Uhr eine verschlossene Glasvitrine, indem er die Glasscheiben mit Hilfe seines Schlüssels (der nicht zum Öffnen der Vitrine bestimmt war) aufhebelte. Die Glasvitrine war mit einer Alarmanlage versehen, die unter anderem Glockengeläut auslöste. T2 entnahm aus dieser Vitrine das „Borghorster Stiftskreuz" und versteckte es unter seinem Pullover. Währenddessen sicherte N1, der zur Eingangstür zurücklief, die Tat ab. Die drei Täter verließen die Kirche einvernehmlich mit dem Stiftskreuz. Hierbei handelten sie in der Absicht, das Kreuz sich oder einem Dritten rechtswidrig zuzueignen. Sie verließen die Kirche durch die einzige nicht abgeschlossene, vom Hauptportal aus gesehen rechte Tür und liefen schnell über den Kirchplatz zurück etwa zu der Stelle, an der sie auch ausgestiegen waren. Der Fahrer des Mercedes fuhr zu diesem Zeitpunkt wieder vor, hielt an und ließ die drei anderweitig Verurteilten wieder einsteigen. Dann flüchteten sie gemeinsam.

[16] Dass der Angeklagte D1 in irgendeiner Weise an diesem Diebstahl beteiligt war, insbesondere, dass er den Diebstahl in Auftrag gab, konnte nicht zur Überzeugung der Kammer festgestellt werden,

[17] Bei dem „Borghorster Stiftskreuz" handelt es sich um ein 41 Zentimeter hohes und 28 Zentimeter breites Kreuz, das einen Holzkern und (nur) auf der Vorderseite ein Goldblech hat. Es sind mehrere Halbedelsteine und kleine Behältnisse eingearbeitet, in denen sich drei christliche Reliquien, sogenannte Berührungsreliquien, befinden. So sollen sich darin ein Holzsplitter des Kreuzes Jesu, ein Teil eines Schwammes Jesu sowie eine Reliquie vom Bett Mariens befinden, wobei die Kammer nicht festgestellt hat, dass es sich tatsächlich um derartige Gegenstände handelt. Das Kreuz stellt ein herausragendes und in seiner Art einzigartiges kunsthistorisches und religiöses Werk des 11. bis 12. Jahrhunderts dar. Der Wert des Kreuzes als kunsthistorisch herausragendes religiöses Kunstwerk beträgt mehrere Millionen Euro. So wurde es bereits auf drei Ausstellungen einmal mit 7,5 Mio. Euro und zweimal mit 7,75 Mio. Euro versichert. Aktuell war es zu einem Wert von 2 Mio. Euro versichert. Das Kreuz diente an dem Standort der Verehrung durch die Gläubigen und wurde außerdem jährlich am 14. September zur Kreuzerhöhung im Gottesdienst verwendet.

[18] Der reine Materialwert konnte von der Kammer nicht genau festgestellt werden. Jedenfalls übersteigt der Versicherungswert den Materialwert des Kreuzes um ein Vielfaches.
[19] 2.

[20] Nach dem Diebstahl behielten die drei anderweitig Verurteilten das Kreuz zunächst bei sich; abredegemäß versteckte es der Zeuge N1 in seiner Nachbarschaft an einem nicht näher bekannten Ort.

[21] Bereits kurz nach der Tat wurden Lichtbildaufnahmen der anderweitig Verurteilten aus den Tankstellen veröffentlicht und eine Öffentlichkeitsfahndung durchgeführt. Dabei wurden die anderweitig Verurteilten auch von Familienmitgliedern und Bekannten aus C2 identifiziert. Aus den Zeitungen erfuhren die Täter von dem hohen ideellen Wert des Stiftskreuzes. Das Bistum Münster setzte für die Wiederbeschaffung des Kreuzes oder zur Ergreifung der Täter eine Belohnung von $50.000 €$ aus.

[22] Möglicherweise versuchten die drei anderweitig Verurteilten erfolglos, das Stiftskreuz gewinnbringend zu verkaufen. Jedenfalls bot der Angeklagte den drei anderweitig Verurteilten in der Folgezeit an, das Stiftskreuz gegen eine entsprechende Bezahlung über einen unbekannten Dritten, den er selbst als "U2" bezeichnet, an die Kirche zurückzugeben, wobei dieser "U2" selbst eine entsprechende Entlohnung der Kirche erwartete, was der Angeklagte wusste. Letztlich willigten die Täter ein, diesem „U2" das Kreuz gegen Zahlung von 150.000 Euro zu überlassen.

[23] Wohl aus Angst vor Fahndungsmaßnahmen beauftragten die drei anderweitig Verurteilten den Zeugen D2, gegen Zahlung einer Belohnung von mindestens 300 Euro bei der Übergabe des Kreuzes an den Angeklagten zu helfen. N1, entweder sein Bruder M2 oder sein Bruder M3, U1, dessen Bruder U2 (genannt S1) und T2 trafen sich mit ihm an einem nicht näher zu ermittelnden Tag im Jahr 2014 - möglicherweise im Februar - an der T3-Tankstelle an der O2-Straße in C2. Dort übergab N1 dem D2 das Stiftskreuz verpackt in einer Tasche. Dieser übernahm die Tasche in dem Wissen, dass sich das Kreuz darin befand, stieg in ein Taxi und brachte es vereinbarungsgemäß zu dem Einkaufszentrum „X2“ in C2. Er lief durch das Einkaufszentrum, wo er vom Angeklagten erwartet wurde. U2 und der Bruder des N1 folgten dem D2 in geringem Abstand, um inn abzusichern. Gemeinsam verließen der Angeklagte und D2 das Einkaufszentrum und begaben sich in ein nahe gelegenes Hotel. Dort suchten sie ein Zimmer auf, wo D2 das Kreuz an den Angeklagten übergab, der es wiederum an einen unbekannten Dritten weiterreichte. Im Gegenzug erhielt der Angeklagte eine Tüte von dem unbekannten Dritten, in der sich mindestens $150.000 €$ Bargeld befanden. Der Angeklagte und D2 verließen das Hotel mit dem Bargeld.

[24] Im Anschluss trafen sich zumindest U1, N1, deren beiden Brüder sowie T2 mit dem Angeklagten in einer Wohnung in C2M4, P. Zumindest die drei anderweitig verurteilten Täter erhielten dort vom Angeklagten jeweils 37.500 Euro ausgezahlt. Was mit den weiteren mindestens 37.500 Euro geschah, blieb unklar. 
Die genauen Umstände dieser Übergabe, insbesondere ob der Angeklagte zuvor noch seine eigene Wohnung mit N1 aufsuchte und dort noch Geld holte, konnten nicht geklärt werden.

[25] Der Angeklagte handelte bei dieser Tat zumindest auch in der Absicht, den Dieben des Kreuzes dessen wirtschaftlichen Wert zu erhalten. Er vermittelte die Weitergabe des Kreuzes in dem Bewusstsein und auch der Absicht, den Dieben eine Gegenleistung für die Rückgabe in Höhe von 150.000 Euro zu verschaffen und ihnen damit den wirtschaftlichen Wert der Sache zu sichern.

[26] Dass der Angeklagte bei dieser Tat selbst eine Entlohnung erhielt, konnte die Kammer nicht feststellen. Der Angeklagte hätte eine Belohnung hierfür jedoch nach eigenen Angaben nicht abgelehnt.

[27] In der Folgezeit gelangte das Stiftskreuz (zunächst) nicht an seinen rechtmäßigen Eigentümer zurück.

[28] Am 00.00.0000 wurden die anderweitig Verurteilten in C2 wegen des Diebstahls des Stiftskreuzes festgenommen. Mit Urteil vom 00.00.0000, rechtskräftig seit dem 00.00.0000, verurteilte das Landgericht Münster wegen dieser Tat T2 und N1 zu einer Freiheitsstrafe von jeweils vier Jahren sechs Monaten und U1 zu einer Freiheitsstrafe von fünf Jahren.

[29] Nach der erstinstanzlichen Verurteilung, aber noch vor Rechtskraft des Urteils, erklärten sich U1 und N1 erstmalig bereit, gegenüber der Polizei Angaben zum Tathergang zu machen. Hierbei belasteten sie den Angeklagten, sie mit dem Diebstahl beauftragt zu haben.

[30] Der Angeklagte wurde aufgrund des Haftbefehls des Amtsgerichts Münster vom 00.00.0000 - 00 Gs 0000/00 - am 00.00.0000 festgenommen und befand sich seit diesem Tag in Untersuchungshaft.

[31] Am 00.00.0000 übergab Rechtsanwalt T4, der Verteidiger des Angeklagten, in C2 dem von der Versicherung des Bistums beauftragten Rechtsanwalt S2 das „Borghorster Stiftskreuz" gegen Zahlung von mindestens 98.000 Euro (vereinbart war eine Summe von 100.000 Euro). Das Stiftskreuz wurde unbeschädigt an das Bistum Münster übergeben.

[32] Durch Beschluss vom 15.2.2017 hat die Kammer den Haftbefehl zunächst gegen Auflagen außer Vollzug gesetzt und letztlich durch Beschluss vom 12.4.2017 aufgehoben.

\section{[33] III.}

[34] Die Feststellungen beruhen auf dem Ergebnis der Beweisaufnahme in der Hauptverhandlung, deren Art und Umfang sich aus dem Sitzungsprotokoll ergeben.
[35] Der Angeklagte hat eine Beteiligung am Diebstahl vom 29.10.2013 bestritten.

[36] Zwar hält die Kammer eine Beteiligung des Angeklagten für möglich. Sie konnte jedoch nicht zur Überzeugung der Kammer festgestellt werden. Insoweit beruhte der Tatverdacht auf den Angaben insbesondere der Zeugen U1 und N1, der Angeklagte habe sie gegen eine versprochene Zahlung von jeweils 1.000 Euro beauftragt, für ihn das Stiftskreuz zu stehlen. Den Angaben der Zeugen zu dieser angeblichen Beauftragung vermochte die Kammer jedoch nicht zu folgen, weil sie widersprüchlich und schon deshalb nicht glaubhaft sind. Auch Aussagekonstanz und Aussagegenese sind mit erheblichen Mängeln behaftet.

[37] Die Feststellungen zum Tathergang am 29.10.2013 beruhen auf den glaubhaften Angaben des Zeugen KHK M5, der die Ermittlungsergebnisse überzeugend und nachvollziehbar dargestellt hat, sowie den insoweit damit übereinstimmenden und deshalb auch glaubhaften Angaben der Zeugen U1 und N1.

[38] Im Übrigen war der Angeklagte geständig.

[39] Hinsichtlich der Feststellungen zur Übergabe des Kreuzes bei dem Einkaufszentrum "X2" in C2 ist die Kammer den diesbezüglich glaubhaften Angaben des Angeklagten gefolgt, die insoweit auch im Wesentlichen mit den Angaben der Zeugen U1, N1 und D2 übereinstimmen.

[40] Die Kammer ist davon überzeugt, dass der Angeklagte mit Begünstigungsabsicht handelte. Er hat glaubhaft angegeben, er habe den Verkauf des Kreuzes gegen Zahlung von 150.000 Euro an den unbekannten „U2" vermittelt in dem Bewusstsein und aufgrund der Verabredung mit ihm, dass dieser das Kreuz an die Kirche gegen Zahlung einer Belohnung aushändigen solle. Er hat dazu weiter angegeben, sein Neffe T2 habe inm gesagt, das Kreuz solle zerstört werden. Er habe gedacht, es sei besser, man bekomme Geld dafür und es gehe zurück. In der Zeitung habe gestanden, dass eine Belohnung von 50.000 Euro ausgesetzt gewesen sei. Die Täter hätten dieses Geld haben wollen. Eine Belohnung für sich selbst - so er sie erhalten hätte - hätte er angenommen. Dass der Angeklagte es offensichtlich dem unbekannten Dritten überließ, ob und wie das Kreuz tatsächlich an den rechtmäßigen Eigentümer zurückkehrte, lässt auf eine relative Gleichgültigkeit hinsichtlich der tatsächlichen Rückgabe schließen. Vielmehr war sein vorrangiges Ziel zumindest auch, den Tätern den wirtschaftlichen Wert der Beute zu sichern.

\section{[41] IV.}

[42] Durch die Vermittlung des Weiterverkaufs hat sich der Angeklagte wegen Begünstigung gemäß § 257 Abs. 1 StGB strafbar gemacht. 


\section{[43] V.}

[44] Soweit die Staatsanwaltschaft dem Angeklagten zur Last gelegt hat, im Oktober 2013 in C2 den Diebstahl des Kreuzes bei den vier Tätern vorab in Auftrag gegeben und ihnen eine Belohnung von jeweils 1.000 Euro in Aussicht gestellt zu haben, war der Angeklagte aus tatsächlichen Gründen freizusprechen. Es konnte nicht zur Überzeugung der Kammer festgestellt werden, dass der Angeklagte D1 in irgendeiner Weise an diesem Diebstahl beteiligt war.

\section{[45] VI.}

[46] Ausgehend vom Strafrahmen des § 257 Abs. 1 StGB hat die Kammer unter Abwägung aller für und gegen den Angeklagten sprechenden Umstände und dabei insbesondere auch unter Berücksichtigung des hohen Wertes des Stiftskreuzes auf eine Freiheitsstrafe von

[47] einem Jahr neun Monaten

[48] als tat- und schuldangemessen erkannt.

\section{[49] VII.}

[50] Die Vollstreckung der Strafe konnte gemäß § 56 StGB zur Bewährung ausgesetzt werden. Insbesondere liegen bei dem Angeklagten besondere Umstände im Sinne des § 56 Abs. 2 StGB vor, obwohl er bereits vorbestraft ist. Diese Vorstrafen liegen einige Jahre zurück. Die Aussetzungen der Reststrafen zur Bewährung wurden nicht widerrufen. Der Angeklagte hat während der Hauptverhandlung wesentlich zur Aufklärung der Tat beigetragen, indem er sie - soweit er verurteilt wurde - gestanden hat. Er hat feste soziale Bindungen und eine Arbeitsstelle.

[51] Aufgrund dieser Umstände geht die Kammer auch von einer günstigen Sozialprognose im Sinne des § 56 Abs. 1 StGB aus und hegt die Erwartung, dass der Angeklagte sich schon die Verurteilung zur Warnung dienen lassen und künftig auch ohne die Einwirkung des Strafvollzugs keine Straftaten mehr begehen wird.

\section{[52] VIII.}

[53] Da nicht festzustellen ist, dass der Angeklagte etwas aus der Tat oder für die Tat erlangt hat, kam die Anordnung des Verfalls nach § 73 StGB bzw. des Verfalls von Wertersatz gemäß § 73a StGB nicht in Betracht.

\section{[54] IX.}

[55] Von einer Entscheidung über den Adhäsionsantrag hat die Kammer abgesehen, weil der Antrag unzulässig ist. Er entspricht nicht den inhaltlichen Anforderungen des $\S 404$ Abs. 1 S. 2 StPO. Nach dieser Vorschrift muss der Antrag den Gegenstand und den Grund des Anspruchs bestimmt bezeichnen und soll die Beweismittel enthalten. Zum Grund des Anspruchs gehören alle Tatsachen, die den Antrag schlüssig machen (Meyer-Goßner in: Meyer-Goßner/Schmitt, StPO, 59. Aufl. 2016, § 404 Rn. 3). Daran fehlt es hier. Denn im Antrag wird bereits der ihm zugrunde liegende Sachverhalt nicht ausreichend dargelegt. So fehlen insbesondere Angaben dazu, aufgrund welcher Tatsachen der Angeklagte gegenüber dem Bistum haften soll und weshalb der (behauptete) Wert des Stiftkreuzes der Gebührenberechnung zugrunde gelegt werden soll. Die mangelhafte Begründung des Adhäsionsantrags führt nach $\S 406$ Abs. 1 S. 3 StPO zu seiner Unzulässigkeit mit der Folge, dass die Kammer von einer Entscheidung abzusehen hatte (vgl. auch Meyer-Goßner in: Meyer-Goßner/Schmitt, StPO, 59. Aufl. 2016, § 406 Rn. 10).

\section{[56] X.}

[57] Die Kostenentscheidung hinsichtlich des Adhäsionsantrags beruht auf $\S 472 a$ Abs. 2 StPO. Danach hat die Kammer die durch das Adhäsionsverfahren entstandenen gerichtlichen Auslagen der Staatskasse auferlegt. Die sonstigen durch dieses Verfahren entstandenen Auslagen hat jeder Beteiligte selbst zu tragen. Dabei hat die Kammer unter Ausübung des ihr zuerkannten pflichtgemäßen Ermessens berücksichtigt, dass von einer Entscheidung über den Adhäsionsantrag (nur) mangels Zulässigkeit abgesehen wurde, obwohl dem Grunde nach ein Schadensersatzanspruch des Bistums gegen den Angeklagten wegen seiner Beteiligung an dem Weiterverkauf des Kreuzes an den unbekannten Dritten in Betracht kommen dürfte.

[58] Im Übrigen folgt die Kostenentscheidung aus $\S 467$ Abs. 1 StPO. Dabei hat die Kammer von der Möglichkeit, die Kosten nach Bruchteilen zu verteilen ( $\$ 464 d$ StPO) nach Ausübung pflichtgemäßen Ermessens keinen Gebrauch gemacht, weil es sachgerechter erscheint, es bei der Regelung des $\S 467$ Abs. 1 StPO zu belassen. 